\title{
HÁBITOS ALIMENTARES, ESTADO NU- TRICIONAL E ESTILOS DE VIDA DE UMA AMOSTRA DE TIMORENSES RESIDENTES EM PORTUGAL
}

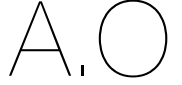

ARTIGO ORIGINAL

${ }^{1}$ Faculdade de Ciências da Nutrição e Alimentação da Universidade do Porto, Rua do Campo Alegre,

n. ${ }^{\circ} 823$,

4150-180 Porto, Portugal

2 Centro de Reabilitação da Associação do Porto da Paralisia Cerebral,

Rua Delfim Maia, $n .^{\circ} 276$,

4200-253 Porto, Portugal

${ }^{3}$ Laboratory for Integrative and Translational Research in Population Health (ITR), Rua das Taipas, n. ${ }^{\circ} 135$, 4050-600 Porto, Portugal

4 GreenUPorto -

Sustainable Agrifood Production Research Centre,

Edifício de Ciências

Agrárias (FCV2),

Rua da Agrária, n. ${ }^{\circ} 747$,

4485-646 Vairão, Portugal

Endereço para correspondência:

Cláudia Afonso

Faculdade de Ciências da

Nutrição e Alimentação da

Universidade do Porto,

Rua do Campo Alegre, n. 823

4150-180 Porto, Portugal

claudiaafonso@fcna.up.pt

Histórico do artigo:

Recebido a 29 de outubro de 2021 Aceite a 31 de dezembro de 202

\section{EATING HABITS, NUTRITIONAL STATUS AND LIFESTYLES IN A TIMORESE SAMPLE LIVING IN PORTUGAL}

Renilde Monteiro' iD ; Rui Poínhos' iD ; Maria Antónia Campos² iD ; Beatriz Teixeira 1,3 iD ; Cláudia Afonso ${ }^{1,3,4^{*}}$ iD

\section{RESUMO}

INTRODUÇÃo: A presença de um número considerável de timorenses a residir em Portugal, justifica a necessidade de investigar acerca dos seus hábitos alimentares, uma vez que até à data, existe uma lacuna de informação relativamente a este tema.

OBJETIVOS: Avaliar e conhecer os hábitos alimentares, o estado nutricional e os estilos de vida de uma amostra de timorenses a residir em Portugal.

METODOLOGIA: Trata-se de um estudo epidemiológico descritivo observacional, de desenho transversal que abrange uma amostra da população timorense residente em Portugal, de idade igual ou superior a 18 anos. Integraram a amostra 88 indivíduos. Foi elaborado um questionário estruturado de aplicação direta, com questões relativas às características sociodemográficas, estilos de vida e estado de saúde, caraterísticas antropométricas, hábitos alimentares e suas alterações após a emigração. A recolha de dados aconteceu entre fevereiro e março de 2020.

RESULTADOS: Foram analisadas 82 respostas dos participantes com idade média de 29,7 $\pm 12,5$ anos. A maioria apresentava um Índice de Massa Corporal normal (64,1\%) e 21,9\% eram pré-obesos ou obesos. 26,5\% dos inquiridos realizaram as refeições, de forma completa e saudável. 62,2\% da amostra relatou melhorias no seu estado de saúde após a vinda para Portugal e 69,5\% considerou que a alimentação teve influência nessa mudança. Relativamente às alterações dos hábitos alimentares, $70,7 \%$ dos inquiridos mencionaram terem alterado o número de refeições, as quantidades de alimentos consumidos (78\%) e 58,5\% o local habitual onde realizavam as suas refeições.

CONCLUSÕES: Dos timorenses que vieram para Portugal, só 1/4 da amostra realizaram refeições completas e saudáveis, mas só cerca de metade ingeria frutas e hortícolas. Com a vinda para Portugal, reportaram consumir um maior número de refeições, maiores quantidades de alimentos e faziam as suas refeições mais vezes fora de casa. Reduziram a prática de atividade física e $1 / 4$ da amostra fumava e bebia álcool semanalmente. Mais de metade da amostra era normoponderal, apresentando pré-obesidade e obesidade $1 / 4$ dos inquiridos.

\section{PALAVRAS-CHAVE}

Alimentação, Imigração, Malnutrição, Portugal, Timor

ABSTRACT

INTRODUCTION: A considerable number of Timorese living in Portugal justifies the need to investigate their eating habits, since to date there is a lack of information on this topic.

OBJETIVES: To evaluate the eating habits, nutritional status and lifestyles from a sample of the Timorese population living in Portugal. METHODOLOGY: This is a cross-sectional design, covering a sample of the Timorese population living in Portugal, aged 18 years or above. The sample comprised 88 individuals. A structured questionnaire for direct application was prepared, with questions related to sociodemographic characteristics, lifestyles and health status, anthropometric characteristics, eating habits and their changes after emigration. Data collection took place between February and March 2020.

RESULTS: The sample include 82 responses from Timorese participants (mean age of 30 years, SD = 12). Most of them had a Body Mass Index corresponding to normality (64.1\%) and 21.9\% were pre-obese or obese. $26.5 \%$ of the participants ate their meals in a complete and healthy way. $62.2 \%$ of the sample reported improvements in their health status after coming to Portugal and $69.5 \%$, considered that diet had an influence on this change. Regarding changes in EA, 70.7\% of the respondents mentioned having changed the number of meals, $78.0 \%$ the quantities of food consumed and $58.5 \%$ the place where they usually ate. CONCLUSIONS: Among this sample of Timorese who came to Portugal, only one quarter of the sample had complete and healthy meals, and only half of them ate fruits and vegetables. Upon coming to Portugal, they reported an increasing of the number of meals, greater amounts of food and eating out more often. They reduced the practice of physical activity and one quarter of the sample smoked and drank alcohol weekly, with the number of hours of sleep within the recommended range. More than half of the sample had normal weight, with pre-obesity or obesity being present in about one quarter.

KEYWORDS

Eating habits, Immigration, Malnutrition, Portugal, Timorese 


\section{INTRODUÇÃO}

A República Democrática de Timor Leste ou Timor-Leste (TL), localizado no Sudoeste Asiático têm aproximadamente 1,3 milhões de habitantes (1). Apesar da percentagem da população que vivia no limiar da pobreza ter decrescido (de 50,4\% em 2007 para 41,8\% em 2014) este valor é ainda elevado (2). O grande desafio de saúde pública que Timor enfrenta é a desnutrição (3). Um problema grave com consequências diversas, interferindo com o comprometimento do desempenho escolar e da produtividade das crianças e, em situações mais graves, a morte precoce (4). Embora o país, com o apoio das organizações nacionais e internacionais, tenha estabelecido políticas e estratégias relacionadas com alimentação/nutrição de forma a combater este flagelo (5-8), a prevalência de nanismo nas crianças com idade inferior a 5 anos continua alta $(49,2 \%)$ (2), tendo-se também verificado o aumento da prevalência de crianças com excesso de peso em cerca de $6 \%$ (9). Nos indivíduos com idades compreendidas entre os 15 e os 49 anos a prevalência de baixo peso nas mulheres é de $27 \%$ e de $26 \%$ nos homens. Por outro lado, $8 \%$ das mulheres e $5 \%$ nos homens têm excesso de peso (9).

Os alimentos-base para esta população são o milho, o arroz, a mandioca e vegetais. O consumo de alimentos de origem animal é baixo $(8,10$, 11). A alimentação dos timorenses é carente e pouco diversificada quanto aos alimentos consumidos, tendo como consequência uma ingestão calórica também inferior às necessidades $(4,8)$.

Em Portugal dados recentes, apontam que existem cerca de 477 mil imigrantes com estatuto legal de residente no país (12). O número dos timorenses registado nos Serviços de Estrangeiros e Fronteiras do ano 2018 foi de 263 (13). São vários os fatores que podem afetar a saúde de um imigrante, sendo um deles a mudança dos hábitos alimentares (HA) (14). Tanto quanto é do nosso conhecimento, até à data, ainda não se encontram dados que relatem os HA dos timorenses em Portugal. Desta forma, considerou-se pertinente estudar os HA e suas alterações nesta população.

\section{OBJETIVOS}

O presente estudo tem como finalidade caracterizar os $\mathrm{HA}$, o estado nutricional e os estilos de vida de uma amostra da população timorense residente em Portugal.

\section{METODOLOGIA}

\section{População e Amostra}

Trata-se de um estudo epidemiológico descritivo observacional, de desenho transversal que abrange uma amostra da população timorense residente em Portugal, de idade igual ou superior a 18 anos. Dos 131 timorenses contactados, 88 responderam ao questionário. Destas, 6 respostas foram excluídas por não responderem de forma completa ao questionário. Trata-se de uma amostra recolhida através do método de amostragem em bola de neve.

\section{Métodos}

De modo a cumprir os objetivos delineados elaborou-se um questionário estruturado de aplicação direta

Previamente à submissão da proposta de trabalho à Comissão de Ética da Faculdade de Ciências da Nutrição e Alimentação da Universidade do Porto foi realizado um estudo piloto em 4 adultos.

Foi solicitada e obtida a colaboração por parte do Presidente do Grupo dos Estudantes Timorenses no Porto, de forma a auxiliar à divulgação deste trabalho aos timorenses residentes neste local. De forma a aumentar a amostra do presente estudo, foi pedido aos potenciais participantes, a disponibilização de possíveis contactos de outros timorenses que poderiam eventualmente participar neste estudo.

Relativamente aos timorenses que não residiam no Porto, foi feita uma pesquisa pela investigadora principal através das redes sociais e posteriormente a mesma entrou em contacto direto, de forma a solicitar o preenchimento de toda a documentação necessária, concretamente o termo do consentimento informado e o link do questionário (na versão Google Forms da Universidade do Porto) de forma a facilitar o seu preenchimento.

O trabalho foi submetido e aprovado pela Comissão de Ética da Faculdade de Ciências da Nutrição e Alimentação da Universidade do Porto (PARECER No2/2020/CEFCNAUP/2020).

\section{Questionário}

O questionário foi adaptado do trabalho de investigação de Sorokina (15) e é constituído pela seguinte informação: características sociodemográficas; estilos de vida (prática de atividade física, consumo de álcool, tabaco e n. ${ }^{\circ}$ de horas de sono) e estado de saúde declarado pelo próprio; caraterísticas antropométricas (peso e estatura autorreportados) e avaliação do consumo alimentar na qual se utilizou o questionário às 24 horas anteriores. Para a classificação das refeições, provenientes da informação supracitada, em completa e incompleta, os alimentos foram agrupados tendo sido considerandos os critérios apresentados na Tabela 1. Paralelamente, foram identificados nas refeições acima mencionadas, a presença ou não de alimentos não recomendados conforme os critérios apresentados na Tabela 2, que permitiram classificar a refeição em saudável ou não. O questionário contém ainda questões sobre as alterações dos HA identificadas após a emigração.

\section{Recolha de Dados}

A recolha dos dados foi efetuada de duas formas distintas: para os residentes no Porto, foi feita de forma presencial num local acordado antecipadamente com uma investigadora e, para os não residentes no Porto o questionário foi reproduzido no formato Google Forms da Universidade do Porto e enviado o link por correio eletrónico para o seu preenchimento. Para as duas situações, apenas foi entregue e enviado o questionário aos participantes que apresentaram previamente o termo do consentimento devidamente assinado.

\section{Tabela 1}

Classificação das refeições de acordo com a sua composição

\begin{tabular}{|c|c|c|}
\hline REFEIÇÕES INTERMÉDIAS & $\begin{array}{c}\text { COMPLETA } \\
\text { N. }{ }^{\circ} \text { DE COMPONENTES* }\end{array}$ & $\begin{array}{c}\text { INCOMPLETA } \\
\text { N. }{ }^{\circ} \text { DE COMPONENTES* }\end{array}$ \\
\hline Pequeno-almoço & 3 & $\leq 2$ \\
\hline Lanche de manhã e tarde & $\geq 2$ & $<2$ \\
\hline Ceia & $\geq 1$ & 0 \\
\hline REFEIÇÕES PRINCIPAIS & $\begin{array}{c}\text { COMPLETA } \\
\text { N. }{ }^{\circ} \text { DE COMPONENTES }{ }^{* \star}\end{array}$ & $\begin{array}{c}\text { INCOMPLETA } \\
\text { N.* DE COMPONENTES** }\end{array}$ \\
\hline Almoço e Jantar & $\geq 4$ & $<4$ \\
\hline
\end{tabular}

*Componentes: laticínios; cereais e derivados; fruta

${ }^{\star}$ Componentes: sopa ou hortícolas no prato; arroz, massa ou batata; carne, peixe, ovo e/ou leguminosas; fruta 
Tabela 2

Classificação referente à presença de alimentos não recomendáveis

\begin{tabular}{|c|c|}
\hline REFEIÇÕES & ALIMENTOS NÃO RECOMENDÁVEIS \\
\hline \multirow{3}{*}{ Intermédias } & 1. Produtos açucarados/Doces/... \\
\hline & 2. Folhados/Fritos/Salgados/... \\
\hline & 3. Presença de ambos \\
\hline \multirow{5}{*}{ Principais } & 1. Açucarados/Doces \\
\hline & 2. Folhados/Fritos \\
\hline & 3. Salgados \\
\hline & 4. Presença de dois destes alimentos \\
\hline & 5. Presença de três destes alimentos \\
\hline
\end{tabular}

\section{Análise Estatística dos Dados}

A análise estatística dos dados recolhidos foi realizada no programa Statistical Package for Social Sciences, versão 26.0. A estatística descritiva consistiu no cálculo de frequências absolutas ( $n$ ) e relativas (\%), de médias e desvios padrão, e de medianas e percentis (P25; P75). Foi utilizada a correlação de Spearman (rs) para medir a associação entre pares de variáveis.

\section{RESULTADOS}

\section{Caraterização da Amostra}

Foram analisadas 82 respostas, das quais $53,7 \%$, são de sexo feminino. A idade média dos participantes foi de 29,7 $\pm 12,5$ anos. $58,5 \%$ dos inquiridos habitavam na região Norte do país, $23,2 \%$ no Centro e os restantes 18,3\% residem no Sul do país e nos Açores. $78 \%$ dos inquiridos frequentavam o ensino superior. A maioria dos participantes (82,9\%) eram estudantes e apenas $22 \%$ eram casados. $O$ tempo de permanência dos inquiridos em Portugal variava entre menos de 1 e 45 anos, com uma média de 5,4 $\pm 8,0$ anos. Relativamente ao grau de adaptação a Portugal, $39 \%$ dos inquiridos referiam sentirem-se muito adaptados (5, numa escala de 1 a 5 ) e apenas $2,4 \%$ relataram o oposto (nível 1).

\section{Caraterização dos Estilos de Vida e Estado Nutricional}

Mais de metade dos inquiridos $(62,2 \%)$ não praticava atividade física (AF). Dos que praticavam (37,8\%), em média faziam-no três vezes

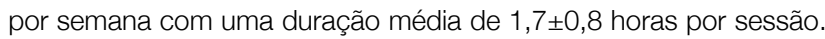
Antes de emigrar, 45,1\% encontrava-se envolvido na prática de AF, com frequência e duração média por sessão, idênticas a quem pratica atualmente. 20,7\% dos inquiridos fumam, sendo a quantidade média de cigarros de $8,0 \pm 7,9$ por dia. No que diz respeito ao consumo de bebidas alcoólicas, $68,3 \%$ dos inquiridos referiam consumir, sendo que a quantidade de bebidas consumidas variava entre 1 ou mais de 2 copos por cada ingestão. Quanto à regularidade de consumo 35,4\% referiam um consumo ocasional e 20,7\% semanal. Relataram a presença de doenças $18,3 \%$ dos participantes, sendo as mais frequentes a diabetes, doença cardiovascular, hipertensão arterial, depressão, cancro, gota, dislipidemia, problemas ósseos e de estômago. A média do número de horas de sono indicada pelos inquiridos foi de $7,2 \pm 1,7$ horas por noite, com um mínimo de 4 horas $e$ um máximo de 12 horas. O peso que reportaram oscilou entre os $42 \mathrm{e}$ $90 \mathrm{~kg}$, e a altura entre 145 e $183 \mathrm{~cm}$, o que correspondeu a uma média de Índice de Massa Corporal (IMC) de 22,5 $\pm 3,8 \mathrm{~kg} / \mathrm{m}^{2}$. De uma forma geral, a percentagem dos participantes com o IMC correspondente à normalidade foi superior comparativamente às demais categorias de IMC (64,1\%). Cerca de 21,9\% dos inquiridos tinha excesso de peso.

\section{Avaliação dos Hábitos Alimentares}

De um modo geral, no que diz respeito à presença de alimentos não recomendáveis nas refeições, verificou-se que os alimentos que se encontram nas refeições intermédias são os produtos alimentares açucarados e/ou folhados. Já nas refeições principais, para além dos produtos alimentares referidos anteriormente, foram ainda identificados a presença de alimentos fritos e/ou salgados e fast-food. No que se refere ao consumo de frutas, $57,3 \%$ dos participantes incluía este alimento ao almoço, 52,4\% ao jantar, 31,7\% ao pequeno-almoço, $19,5 \%$ a meio de manhã, $28 \%$ a meio da tarde, e apenas $9,8 \%$ dos participantes a ceia. Os inquiridos que consumiam hortícolas como acompanhamento do prato ao almoço e jantar eram 57,3\% e 50\%, respetivamente.

$\mathrm{Na}$ Tabela 3 encontram-se apresentadas informações referentes à frequência do consumo das diferentes refeições, à sua constituição (completa ou não), à presença de alimentos não recomendáveis (refeição saudável ou não), e se a refeição era completa e simultaneamente saudável.

\section{Alterações Autorreportadas Decorridas com a Vinda para Portugal}

Relativamente aos estilos de vida, identificou-se que $7,3 \%$ dos indivíduos deixaram de realizar AF. 58,5\% dos inquiridos aumentaram de peso depois de emigrar. Para caraterizar o seu estado de saúde em Portugal em comparação com o tempo em que viviam em Timor (numa escala de 1 a 5 em que 1 significa piorou muito e 5 melhorou muito) verificou-se que $62,2 \%$ dos inquiridos indicaram melhorias no seu estado de saúde após a sua vinda para Portugal (correspondendo à junção dos níveis 4 e 5). Quanto questionados sobre se consideravam que a alimentação teve alguma influência nessa mudança do seu

\section{Tabela 3}

Inquiridos que realizaram as refeições, a sua composição (refeições completas ou não), presença de alimentos não recomendáveis (refeição saudável ou não) e refeição completa e saudável, nas 24 horas anteriores

\begin{tabular}{|c|c|c|c|c|}
\hline REFEIÇÕES & $\begin{array}{l}\text { INQUIRIDOS QUE } \\
\text { REALIZARAM A REFEIÇÃO } \\
\mathrm{n}(\%)\end{array}$ & $\begin{array}{l}\text { INQUIRIDOS QUE REALIZARAM } \\
\text { UMA REFEICCÃO COMPLETA** } \\
n(\%)\end{array}$ & $\begin{array}{l}\text { PRESENÇA DE ALIMENTOS NÃO } \\
\text { RECOMENDÁVEIS NA REFEIÇÃO* } \\
\text { n (\%) }\end{array}$ & $\begin{array}{l}\text { INQUIRIDOS QUE REALIZARAM UMA } \\
\text { REFEIÇÃO COMPLETA E SAUDÁVEL* } \\
\text { (SEM ALIMENTOS NÃO } \\
\text { RECOMENDÁVEIS) }\end{array}$ \\
\hline Pequeno-Almoço & $72(87,8 \%)$ & $26(36,1 \%)$ & $38(52,8 \%)$ & $9(12,5 \%)$ \\
\hline Merenda da manhã & $28(34,1 \%)$ & $15(53,6 \%)$ & $13(46,4 \%)$ & $6(21,4 \%)$ \\
\hline Almoço & $82(100 \%)$ & $44(53,7 \%)$ & $48(58,5 \%)$ & $17(20,7 \%)$ \\
\hline Merenda da tarde & $58(70,7 \%)$ & $45(77,6 \%)$ & $30(51,7 \%)$ & $23(39,7 \%)$ \\
\hline Jantar & $80(97,6 \%)$ & $29(36,3 \%)$ & $47(58,7 \%)$ & $10(12,5 \%)$ \\
\hline Ceia & $25(30,5 \%)$ & $23(92,0 \%)$ & $10(40,0 \%)$ & $13(52,0 \%)$ \\
\hline Média do total das refeições & $70,11 \%$ & $58,21 \%$ & $51,35 \%$ & $26,46 \%$ \\
\hline
\end{tabular}

*Percentagens calculadas em função do número de inquiridos que realizaram a refeição. 
estado de saúde, e utilizando a escala usada anteriormente, 69,5\%, assinalaram igualmente os níveis 4 e 5. Quando correlacionadas estas duas variáveis, identificou-se que os participantes que consideraram ter melhorado o seu estado de saúde, associaram maioritariamente esta melhoria à influência da alimentação. ( $p<0,001$; $r s=0,434)$. Relativamente às alterações dos HA, $70,7 \%$ dos inquiridos mencionaram ter alterado o número de refeições desde a vinda para Portugal sendo que destes $74,1 \%$ referiram ter aumentado o número das refeições. Por outro lado, $78,0 \%$ dos participantes referiram terem alterado as quantidades de alimentos ingeridos, sendo que de uma forma geral a ingestão tem aumentado. No que diz respeito ao local das refeições $58,5 \%$ indicaram que modificaram o local em que habitualmente realizavam as suas refeições, sendo que após a vinda para Portugal $66,7 \%$ destes faziam as suas refeições mais vezes fora da casa.

\section{DISCUSSÃO DE RESULTADOS}

Ainda que alguns trabalhos de investigação demonstrem que os imigrantes apresentam uma menor prevalência de excesso de peso ou obesidade comparativamente a população hospedeira dos países industrializados (16), outros estudos porém retratam o oposto, sugerindo que os imigrantes são mais predispostos a adquirirem HA nem sempre favoráveis (14), adaptando-se a ambientes obesogénicos $(17,18)$, e consequentemente mais propensos a um aumento da prevalência de fatores de risco de doenças crónicas não transmissíveis (19). No presente estudo $58 \%$ dos participantes reportaram terem aumentado o seu peso, resultados concordantes com trabalhos realizados com imigrantes chineses, ucranianos e turcos residentes em Portugal $(15,20,21)$, ainda que o IMC médio seja de 22,5 5 3,8 $\mathrm{kg} / \mathrm{m}^{2}$ neste grupo de população. No que se refere aos estilos de vida promotores de saúde, a atividade física é dos mais descritos com uma associação positiva (22), e as recomendações da Organização Mundial da Saúde sugerem uma prática regular de 150 minutos de exercícios de intensidade moderada, ou equivalentes ao longo da semana para a população adulta (23). Os resultados obtidos no presente estudo mostram que, com a vinda para Portugal, a prática de atividade física diminuiu 7,3\%. O número médio de horas de sono na população estudada foi de $7,2 \pm 1,7 \mathrm{~h}$, o que se encontra dentro das recomendações sugeridas pelo National Sleep Foundation, ainda próximo do seu limite inferior (24). Com a vinda para Portugal, mais de metade dos participantes da nossa amostra assinalaram terem aumentando o número das refeições por dia, assim como a quantidade dos alimentos consumidos. No que diz respeito aos hábitos alimentares, a maioria dos participantes não realizou uma alimentação nem completa nem saudável nas diferentes refeições. Cerca de metade dos inquiridos incluíam nas suas refeições um ou mais alimentos processados, tais como alimentos fritos e salgados (como batatas fritas, presunto), alimentos ricos em açucares simples (como refrigerantes, sobremesas doces) e gorduras saturadas (como produtos da pastelaria, fast-food). Estes resultados estão em concordância com outros estudos efetuados em populações de países em desenvolvimento que emigraram para países desenvolvidos, concretamente no que se refere ao aumento do consumo de alimentos processados $(25,26)$. É de conhecimento geral que o consumo de frutas e hortícolas é um indicador importante na qualidade alimentar (27), sendo um fator protetor contra doenças crónicas e cancro (28, 29). Uma investigação realizada em imigrantes que viviam em Portugal mostrou que este grupo da população não ingeria uma quantidade adequada deste grupo de alimentos (30). Embora, os resultados do presente trabalho não nos permitam aferir quantidades médias de consumo de fruta, permitem aferir que só cerca de metade dos indivíduos consumia fruta.

Tal como qualquer outro estudo, o presente trabalho apresenta limitações, tais como o seu carácter transversal não permitindo inferir a causalidade entre as variáveis, ainda que esta limitação seja uma consequência do desenho do estudo que em nada interfere com a resposta aos objetivos do mesmo.

Apresentam-se como limitações o tamanho da amostra de conveniência não ser de grande amplitude, assim como o facto de não ter sido recolhida de maneira uniforme (presencialmente e remotamente), porém o investigador principal conseguiu assegurar em qualquer formato de recolha, o esclarecimento de dúvidas aos participantes, no momento do preenchimento do questionário. Quanto à informação sobre o consumo alimentar, poderá ter sido afetada pela desejabilidade social podendo ter ocorrido uma subestimação ou sobrestimação deste consumo, no geral e/ou para grupos específicos de alimentos, associado a qualquer método de avaliação do consumo alimentar no geral e ao questionário às 24 horas anteriores em particular.

O facto de só ter sido avaliado o dia anterior à recolha de dados que poderá não ser representativa do todo, porém por motivos logísticos não foi possível considerar pelo menos o reporte de mais um dia não consecutivo. O facto de termos uma questão sobre se o dia reportado era dia de consumo habitual, o que o foi em $100 \%$ da amostra, consideramos que poderá minorar as consequências deste viés. Também o peso e a altura foram autorreportados, pelo que a prevalência de pré-obesidade e obesidade pode estar subestimada. Apresentam-se como pontos fortes do presente trabalho o seu carácter inovador, uma vez que em Portugal não encontramos trabalhos com os objetivos idênticos realizados nesta população.

\section{CONCLUSÕES}

Os timorenses que vieram para Portugal reportaram uma diminuição da prática de atividade física, cerca de $1 / 4$ da amostra fumava e bebia álcool semanalmente, estando o $\mathrm{n} .^{\circ}$ de horas de sono dentro do recomendado. Mais de metade da amostra era normoponderal, apresentando pré-obesidade e obesidade $1 / 4$ dos inquiridos. Considerando a média de todas as refeições, só $26,5 \%$ da amostra realizaram refeições completas e saudáveis, mas só cerca de metade ingeria frutas e hortícolas. Com a vinda para Portugal, grande parte dos participantes referiram que consumiam um maior número de refeições, maiores quantidades de alimentos e faziam as suas refeições mais vezes fora de casa.

Deste modo, uma adequada intervenção alimentar que articule harmoniosamente os HA do país de origem, com o padrão alimentar Português, alinhado com as recomendações para a prática de uma alimentação saudável, revestem-se de primordial importância para a promoção da saúde nas populações de emigrantes a residirem em Portugal.

\section{REFERÊNCIAS BIBLIOGRÁFICAS}

1. The World Bank. Timor Leste Overwiew Country Profile. The World Bank. 2018. 2. Ministry of Finance of Timor-Leste. Poverty in Timor-Leste 2014. Díli, Timor-Leste: Direção Geral de Estatística de Timor-Leste; 2016.

3. Ministry of Health. Food Based Dietary Guidelines of the Democratic Republic of Timor-Leste. Díli, Timor-Leste: Ministry of Health of Timor-Leste; 2015.

4. Provo A, Atwood S, Sullivan EB, Mbuya N. Malnutrition in Timor-Leste: A review of the burden, drivers, and potential response. 2017.

5. Moriarty H, Darnton-Hill I. Timor-Leste Nutrition Strategic Review. Díli, Timor-Leste: Australian Government's Department of Foreign Affairs and Trade; 2017.

6. Ministry of Health. Timor-Leste National Nutrition Strategy 2014-2019. 2014. 
7. National Council for Food Security Sovereignty and Nutrition in Timor Leste (KONSSANTIL). Zero Hunger Challenge National Action Plan for a Hunger and Malnutrition Free Timor-Leste. Díli. 2014.

8. Government of Democratic Republic of Timor-Leste. National Food and Nutrition Security Policy. Díli, Timor-Leste. 2017.

9. General Directorate of Statistics, Ministry of Health, ICF. Timor-Leste Demographic and Health Survey 2016. Dili, Timor-Leste Rockville, Maryland, USA: GDS and ICF; 2018.

10. Andersen AB, Pant J, Thilsted SH. Food and nutrition security in Timor-Leste. WorldFish. 2013.

11. Wong JT, Bagnol B, Grieve H, da Costa Jong JB, Li M, Alders RG. Factors influencing animal-source food consumption in Timor-Leste. Food Security. 2018;10(3):741-62. 12. Instituto Nacional de Estatística. População estrangeira com estatuto legal de residente: total e por algumas nacionalidades Portugal: INE; 2019 [Available from: https://www.ine.pt/xportal/xmain?xpid=INE\&xpgid=ine_indicadores\&indOcorrCo$d=0009107 \&$ contexto=bd\&selTab=tab2].

13. Serviço de Estrangeiros e Fronteira. Relatório de Imigração, Fronteiras e Asilo 2018. Serviço de Estrangeiros e Fronteira. 2019.

14. Holmboe-Ottesen G, Wandel M. Changes in dietary habits after migration and consequences for health: a focus on South Asians in Europe. Food \& nutrition research. 2012;56.

15. Sorokina A. Avaliação de hábitos alimentares e estado nutricional de uma população ucraniana residente na Zona Norte de Portugal [Tese de Licenciatura]. Porto: Faculdade de Ciências da Nutrição e Alimentação da Universidade do Porto; 2013.

16. McDonald JT, Kennedy S. Is migration to Canada associated with unhealthy weight gain? Overweight and obesity among Canada's immigrants. Social science \& medicine (1982). 2005;61(12):2469-81.

17. da Costa LP, Dias SF, Martins MdRO. Association between length of residence and overweight among adult immigrants in Portugal: A nationwide cross-sectional study. BMC Public Health. 2017;17(1):316.

18. Delavari M, Sønderlund AL, Swinburn B, Mellor D, Renzaho A. Acculturation and obesity among migrant populations in high income countries-a systematic review. J BMC Public Health. 2013;13(1):458.

19. Rechel B, Mladovsky P, Ingleby D, Mackenbach JP, McKee M. Migration and health in an increasingly diverse Europe. The Lancet. 2013;381(9873):1235-45.

20. Li Y. Food habits of a Chinese immigrant population living in Portugal [Tese de Mestrado]. Porto: Faculdade de Ciências da Nutrição e Alimentação da Universidade do Porto; 2015.

21. Akalin FK. Avaliação dos hábitos alimentares de turcos residentes em Portugal [tese de mestrado]. Porto: Faculdade de Ciências da Nutrição e Alimentação da Universidade do Porto; 2016.

22. Penedo FJ, Dahn JR. Exercise and well-being: a review of mental and physical health benefits associated with physical activity. Current opinion in psychiatry. 2005;18(2):189-93.

23. World Health Organization. Global Recommendations on Physical Activity For Health. Geneva, Switzerland: World Health Organization; 2010.

24. Hirshkowitz M, Whiton K, Albert SM, Alessi C, Bruni O, DonCarlos L, et al. National Sleep Foundation's updated sleep duration recommendations: final report. Sleep health. 2015;1(4):233-43

25. Gillbert PA, Khokhar S. Changing dietary habits of ethnic groups in Europe and implications for health. Nutrition reviews. 2008;66(4):203-15.

26. Kumar BN, Holmboe-Ottesen G, Lien N, Wandel M. Ethnic differences in body mass index and associated factors of adolescents from minorities in Oslo, Norway: a cross-sectional study. Public health nutrition. 2004;7(8):999-1008.

27. Gil Á, Martinez de Victoria E, Olza J. Indicators for the evaluation of diet quality. Nutricion hospitalaria. 2015;31 Suppl 3:128-44.

28. World Health Organization. Diet, nutrition and the prevention of chronic diseases. World Health Organization technical report series. 2003;916.

29. World Health Organization. Fruit and Vegetable Promotion Initiative - report of the meeting, Geneva, 25-27 August 2003. Geneva: World Health Organization. 2003.
30. Costa L, Dias S, Martins M. Fruit and Vegetable Consumption among Immigrants in Portugal: A Nationwide Cross-Sectional Study. International journal of environmental research and public health. 2018;15(10). 